\title{
Conductivity of macromolecular networks measured by electrostatic force microscopy
}

\author{
C. H. Lei, ${ }^{\text {a) }}$ A. Das, M. Elliott, and J. E. Macdonald \\ Department of Physics and Astronomy, Cardiff University, Cardiff, P.O.Box 913, CF24 3YB, \\ United Kingdom
}

(Received 11 February 2003; accepted 19 May 2003)

\begin{abstract}
High-resolution electrostatic force microscopy (EFM)-phase measurements are reported on molecular networks of semiconductor polymer poly-hexylthiophene (P3HT) and DNA molecules. A lateral resolution of better than $20 \mathrm{~nm}$ is demonstrated in EFM-phase images of the P3HT network by detecting the phase shift of the tip along the molecules under electrical bias. Strands of $\lambda$-DNA are shown to be highly insulating in comparison to the semiconductor polymer P3HT, with a minimum resistance of $\sim 1 \times 10^{7} \Omega \mathrm{cm}$. (c) 2003 American Institute of Physics.
\end{abstract}

[DOI: $10.1063 / 1.1592888$ ]

There is a continual search for a material systems and fabrication technologies for electrical discrete devices and complex networks, aiming at the smallest possible length scales. Semiconducting conjugated polymers have made rapid progress over recent years for display applications and for electronic devices. For instance, poly-hexylthiophene (P3HT) has been used to make field-effect transistors (FET) devices, and scanning Kelvin probe microscopy has been used to characterize them. ${ }^{1}$ Intense recent effort has focused on exploiting the inherent one-dimensional nature of carbon nanotubes (CNTs) and DNA molecules for devices on the nanometer length scale. FETs have been successfully produced with CNTs. ${ }^{2}$ DNA offers the capability to selfassemble in predetermined structures from strands having engineered base sequences. However, there has been considerable controversy regarding its conductivity; conducting, semiconducting, and insulating behavior have all been recently reported, ${ }^{3-5}$ suggesting that factors such as humidity and adsorbed water might play a significant role. The conductivity of DNA can be modified by doping of the bases, ${ }^{6}$ and metals can be deposited onto the DNA molecules as templates. $^{7}$ Such DNA-based approaches may eventually pave the way to molecular devices of specified architecture. In addition, probing nanometer-scale materials is a challenging task and is a focus of growing attention for the research development. Atomic force microscopy (AFM) techniques hold great promise in this area for providing direct electrical characterization as well as topography. Here, we describe the application of electrostatic force microscopy (EFM), a variant of AFM used to characterize electrical properties of materials. We demonstrate that EFM-phase mode measurements can provide higher lateral resolution measurement than surface potential or scanning Kelvin probe microscopy. In this letter, we apply EFM to the measurement of P3HT and $\lambda$-DNA. P3HT is a conjugated polymer, where $\pi$-electrons are delocalized along the chain, rendering it electrically conductive. The conductivity of P3HT from Sigma-Aldrich is $10^{-6}$ to $10^{-7}$ siemens $/ \mathrm{cm}$. Random P3HT networks are examined, and are believed to result from the interplay of natu-

a)Electronic mail: Leic@cf.ac.uk ral aggregation of molecules, molecule-surface interaction, and the effects of local concentration gradients during growth. We perform electrical measurements of the network with the EFM-phase technique and use it to characterize modifications of the network produced by AFM manipulation of the P3HT molecules. We also apply the measurements to measure the electrical characteristics of partially-aligned $\lambda$-DNA strands, and find insulating behavior.

During an EFM measurement, the main scan records the surface topographical data in tapping mode. In the subsequent interleave scan, the tip is lifted and kept at fixed height (typically $20 \mathrm{~nm}$ ) above the surface, whose topography is obtained in the first scan. During the interleave scan, the tip is kept driven in oscillation with the same driving frequency as in the main scan. The frequency $(\omega)$ or phase $(\phi)$ shift is a function of the surface potential, and are given $b^{8}$

$$
\Delta \omega \approx \frac{\omega_{0}}{2 k} \frac{d F(z)}{d z}, \quad \Delta \phi \approx \frac{Q}{k} \frac{d F(z)}{d z},
$$

where $F(z)$ is the electrostatic force between the tip and surface at the relative separation $z, Q$ is the cantilever quality factor, $k$ is the cantilever spring constant. $F(z)$ is related to the local capacitance $(C)$ between the tip and the sample by

$$
F(z)=\frac{1}{2} \frac{d C}{d z}(\Delta U)^{2}
$$

where $\Delta U$ is the potential difference between the tip and the sample. In the conventional surface potential measurement or Kelvin probe method, ${ }^{9}$ the tip bias is varied to give $F(z)=0$, and hence $\Delta U=0$, so that the tip bias gives a direct measurement of the surface potential. In the EFM-phase method, however, the force gradient rather than the force itself is measured. This provides improved spatial resolution compared with the conventional EFM. ${ }^{10}$ In addition, the cantilever's phase response is more sensitive than its amplitude response to changes in the tip-sample interactions and is less susceptible to height variations on the sample surface.

To prepare the sample, P3HT from Sigma-Aldrich was dissolved in $\mathrm{CCl}_{4}$ and the solution was spin coated onto $\mathrm{SiO}_{2} / \mathrm{Si}$ surfaces to form a network structure. $\lambda$-DNA from 


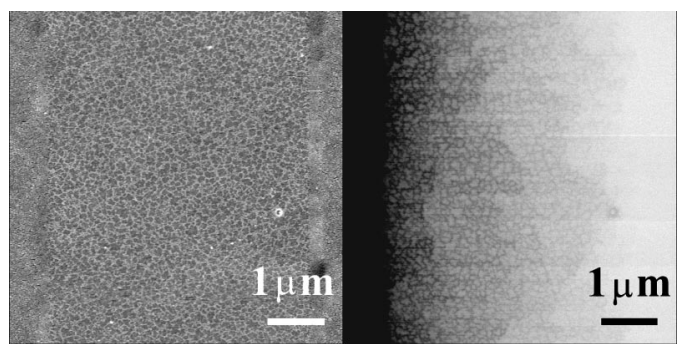

(a)

(b)

FIG. 1. Images of topography and EFM-phase for P3HT with a gold contact at each end. (a) flattened topography image. (b) EFM/phase map when a bias of $+5 \mathrm{~V}$ was applied to the left electrode, the phase range is $5^{\circ}$.

New England Biolabs was first diluted in a $\mathrm{Mg}^{+2}$ buffer, then a drop of this solution was dropped on the $\mathrm{SiO}_{2} / \mathrm{Si}$ surfaces, rinsed with flowing pure water to align the DNA molecules, and dried in flowing compressed nitrogen. After P3HT or DNA deposition, two 15-nm-thick Au electrodes were evaporated onto the sample by a wire shadow mask method. ${ }^{11}$ The wire shadow left a gap of about $4 \mu \mathrm{m}$ on the $\mathrm{Au}$ film. The width of the electrodes is about $3 \mathrm{~mm}$. When making $\mathrm{Au}$ contact by thermal evaporation, care was taken so that organic molecules were kept at a low enough temperature to prevent decomposition, by using a shield to restrict the radiation incident of the sample, and by employing a low gold evaporation rate of $0.1 \mathrm{~nm} / \mathrm{s}$.

Figure 1(a) shows an AFM image of a network structure of P3HT formed on a $\mathrm{SiO}_{2} / \mathrm{Si}$ surface. An $n^{+}$-Si AFM tip with $300-\mathrm{kHz}$ resonant frequency was employed. Its free tapping amplitude was about $13 \mathrm{~nm}$, and the tip was engaged when its amplitude decreased to $85 \%$ of its free amplitude. Similar cellular networks have been reported recently for spin-coated Au nanocrystals decorated with short alkanethiol chains on silicon. ${ }^{12}$ The authors of this study argue that this cellular network cannot be explained based on Marangoni convection alone; instead, spinodal decomposition was found to play key role. It is noteworthy that very similar networks are observed in such different systems as decorated nanoclusters and semiconducting polymer molecules, indicating a universal driving mechanism. Under dc bias, the current passing through this network structure was quite unstable; increasing the film thickness of the P3HT polymer tends to stabilize the current, and $I-V$ measurement in air showed a rectifying behavior of the $\mathrm{Au} / \mathrm{P} 3 \mathrm{HT} / \mathrm{Au}$ system. No current signal could be detected for the insulating substrate $\mathrm{SiO}_{2}$.

Figure 1(b) shows the result of EFM-phase measurements on the $\mathrm{Au} / \mathrm{P} 3 \mathrm{HT} / \mathrm{SiO}_{2}$ sample of Fig. 1(a), obtained by applying a $+5-\mathrm{V}$ dc bias to the left electrode during the interleave scan. The image shows phase shift of the tip during the interleave scan. In contrast, no phase shift was detected on $\mathrm{Au}$ electrodes, $\mathrm{P} 3 \mathrm{HT}$ molecules, or $\mathrm{SiO}_{2}$ substrate when the bias was $0 \mathrm{~V}$. This means that the work function difference between these materials and the tip is not large enough to induce a phase shift on the cantilever. In the EFMphase image, the darker region has a bigger phase lag than the brighter region due to the attractive electrostatic force on the tip, as can be seen in the two Au electrodes on the left and right sides. In the gap region, the P3HT network structure gave a clear phase shift image which coincides with its topographic image, showing a resolution better than $20 \mathrm{~nm}$.

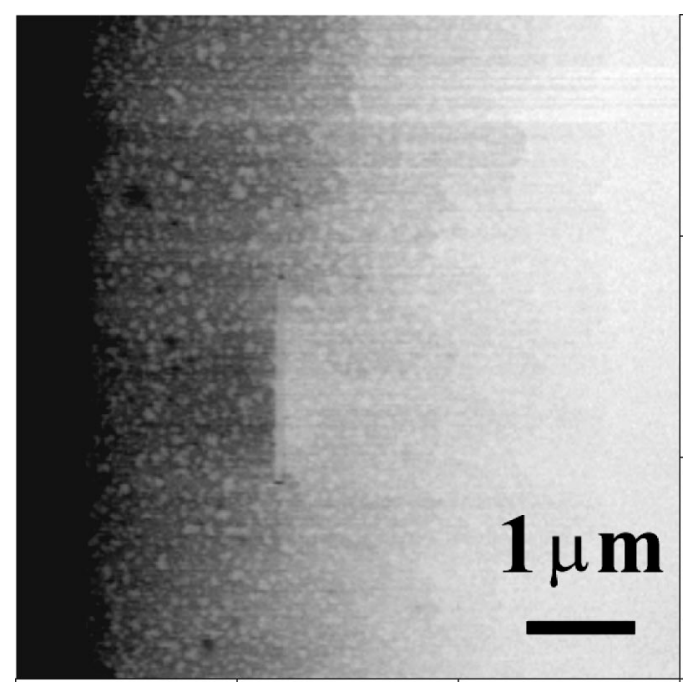

FIG. 2. EFM/phase image of a P3HT network in which a mechanical cut of $2 \mu \mathrm{m}$ has been made with the tip in contact mode. The EFM/phase map was taken under identical conditions to Fig. 1(b) and shows clearly that the molecules immediately to the right of the cut are at a lower potential due to a longer connecting path around the cut. The phase range is $5^{\circ}$.

Since the phase lag decreased gradually from the left side to the right side in the gap region, the potential on the network also decreased gradually, consistent with a conductive network structure. On the other hand, for a clean $\mathrm{SiO}_{2}$ surface, most of the potential is dropped near the edge of the left $\mathrm{Au}$ electrode.

In order to confirm the conducting nature of the P3HT network and the electrostatic origin of the EFM-phase contrast, a $2-\mu \mathrm{m}$ cut on the network along the gap direction was made with the tip, and then EFM-phase measurement was carried out. Figure 2 is the result obtained when applying a $+5-\mathrm{V}$ dc to the left electrode during the lift scan. The cut can be clearly seen in this image, and a potential drop exists across the cut due to the break of electrical connection.

By comparison, a standard surface potential measurement was also carried out on the $\mathrm{Au} / \mathrm{P} 3 \mathrm{HT} / \mathrm{SiO}_{2}$ structure, as shown in Fig. 3, obtained when applying a $+5-\mathrm{V}$ dc bias to the left electrode with the $n^{+}$-Si tip. In the surface potential

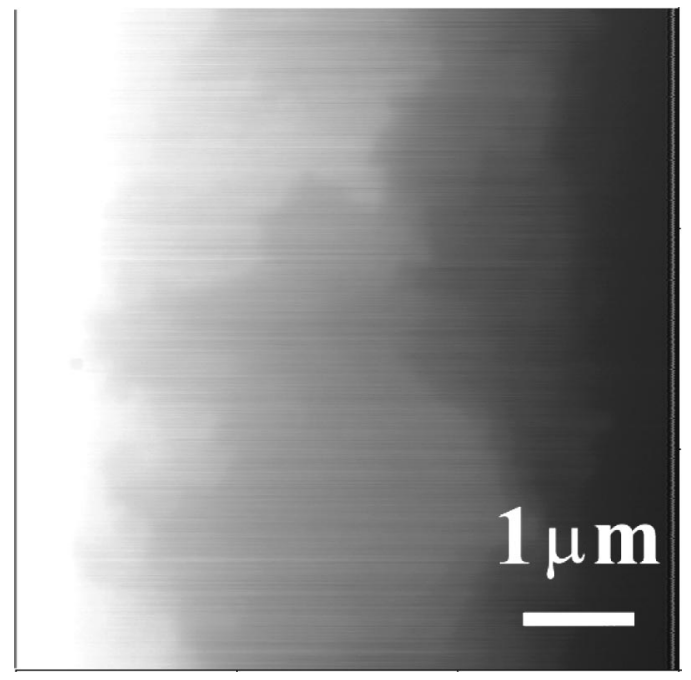

FIG. 3. Conventional EFM surface potential image of the P3HT sample when a bias of $+5 \mathrm{~V}$ was applied to the left electrode. The potential range is $2.5 \mathrm{~V}$. 


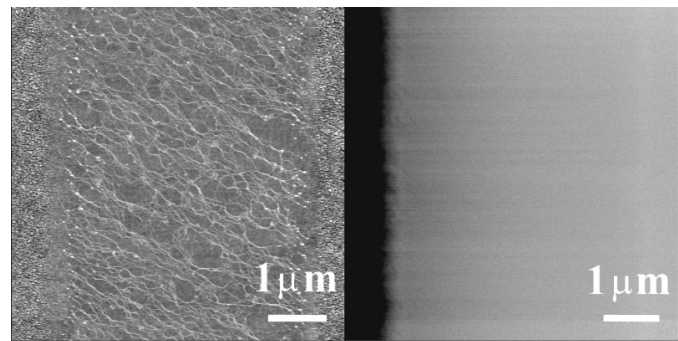

(a)

(b)

FIG. 4. Images of topography and EFM-phase for $\lambda$-DNA. (a) Flattened topography image. (b) EFM/phase map when a bias of $+5 \mathrm{~V}$ was applied to the left electrode. No trace of DNA strands can be found, except a few faint traces near the left Au film edge. The phase range is $5^{\circ}$.

image, the brighter region has a higher potential than the darker region, as can be seen in the two Au electrodes on the left and right sides. In the gap region, the potential decreases gradually from the left side to the right side. Step structures can be seen in the surface potential image, similar to the EFM-phase image in Fig. 1(b). However, the molecular network structure did not appear in the surface potential image. It is clear from this comparison that surface potential measurement has lower lateral resolution than the EFM/phase measurement.

Figure 4(a) is an AFM image of an $\mathrm{Au} / \mathrm{DNA} / \mathrm{SiO}_{2}$ preparation. $\lambda$-DNA strands have been stretched by flowing water and are long enough to bridge the two Au contacts. $I-V$ measurements on this sample gave no measurable current signal $(<1 \mathrm{pA})$. The resistivity of individual $\lambda$-DNA was estimated to be greater than $1 \times 10^{7} \Omega \mathrm{cm}$. Figure $4(\mathrm{~b})$ is an EFM-phase image of Fig. 4(a) with similar $n^{+}$-Si tip, and a $+5-\mathrm{V}$ dc bias was applied to the left Au electrode during the interleave scan. Again, when the bias was $0 \mathrm{~V}$, no phase shift was observed on the sample. In the gap region where DNA lies on the $\mathrm{SiO}_{2}$ surface, although DNA is clearly observed in topography, no trace of DNA strands can be observed in the EFM, except a few faint traces near the left Au film edge.

It is possible that the contact resistance was large enough that almost all the voltage was dropped in the contact region, but this situation did not change with higher biases $(>10 \mathrm{~V})$. DNA molecules in the gap region did not show any signature in the EFM-phase image in comparison to the EFM-phase image of P3HT. This result strongly suggests that the DNA is insulating, as reported by C. Gómez-Navarro $^{13}$ et al., who used similar method to study DNA molecules absorbed on insulators. As outlined earlier, a range of measured conductivities have been reported for DNA from insulating to semiconducting. There is evidence that the conductivity of DNA is affected by humidity. ${ }^{14}$ Pres- ently, our measurements are carried out in air, and the role of humidity in DNA conductivity cannot be confirmed.

In conclusion, EFM has been applied to characterize the electrical behavior of P3HT and DNA molecular networks. The EFM-phase provides a surface potential map of macromolecules in a noncontact mode, with a lateral resolution of $<20 \mathrm{~nm}$, showing the macromolecular conductivity in a relatively straightforward manner. Quantitative analysis is complicated by the actual tip shape and surface topography and is currently being developed. Whereas networks of the semiconducting polymer P3HT are conducting, DNA strands are shown to be fairly highly insulating, with resistivity $\geqslant 1 \times 10^{7} \Omega \mathrm{cm}$. Such scanning-probe-microscopy based techniques are likely to be crucial for characterising, modifying and controlling molecular networks and devices in future nanoelectronics.

The authors would like to thank R. Tucker, I. Robinson, P. Hulyer, and C. Dunscombe in this department for help in sample preparation and technical support, and the EPSRC for financial support.

${ }^{1}$ H. Sirringhaus, P. J. Brown, R. H. Friend, M. M. Nielsen, K. Bechgaard, B. M. W. Langeveld-Voss, A. J. H. Spiering, R. A. J. Janssen, and E. W. Meijer, Synth. Met. 111, 129 (2000); L. Bürgi, H. Sirringhaus, and R. H. Friend, Appl. Phys. Lett. 80, 2913 (2002).

${ }^{2}$ S. J. Wind, J. Appenzeller, R. Martel, V. Derycke, and Ph. Avouris, Appl. Phys. Lett. 80, 3817 (2002).

${ }^{3}$ H.-W. Fink and C. Schönenberger, Nature (London) 398, 407 (1999); A. Yu. Kasumov, M. Kociak, S. Guéron, B. Reulet, V. T. Volkov, D. V. Klinov, and H. Bouchiat, Science 291, 280 (2001).

${ }^{4}$ D. Porath, A. Bezryadin, S. de Vries, and C. Dekker, Nature (London) 403, 635 (2000); L. Cai, H. Tabata, and T. Kawai, Appl. Phys. Lett. 77, 3105 (2000).

${ }^{5}$ P. J. de Pablo, F. Moreno-Herrero, J. Colchero, J. Gómez Herrero, P. Herrero, A. M. Baró, P. Ordejón, J. M. Soler, and E. Artacho, Phys. Rev. Lett. 85, 4992 (2000); A. J. Storm, J. van Noort, S. de Vries, and C. Dekker, Appl. Phys. Lett. 79, 3881 (2001).

${ }^{6}$ A. Rakitin, P. Aich, C. Papadopoulos, Yu. Kobzar, A. S. Vedeneev, J. S. Lee, and J. M. Xu, Phys. Rev. Lett. 86, 3670 (2001).

${ }^{7}$ J. Richter, M. Mertig, W. Pompe, I. Mönch, and H. K. Schackert, Appl. Phys. Lett. 78, 536 (2001).

${ }^{8}$ D. Sarid, Scanning Force Microscopy (Oxford University Press, New York, 1994)

${ }^{9}$ M. Nonnenmacher, M. P. O’Boyle, and H. K. Wickramasinghe, Appl. Phys. Lett. 58, 2921 (1991).

${ }^{10}$ J. Colchero, A. Gil, and A. M. Baró, Phys. Rev. B 64, 245403 (2001).

${ }^{11}$ P. J. de Pablo, E. Graugnard, B. Walsh, R. P. Andres, S. Datta, and R. Reifenberger, Appl. Phys. Lett. 74, 323 (1999).

${ }^{12}$ P. Moriarty, M. D. R. Taylor, and M. Brust, Phys. Rev. Lett. 89, 248303 (2002).

${ }^{13}$ C. Gómez-Navarro, F. Moreno-Herrero, P. J. de Pablo, J. Colchero, J. Gómez-Herrero, and A. M. Baró, Proc. Natl. Acad. Sci. U.S.A. 99, 8484 (2002).

${ }^{14}$ Y. Otsuka, H. Lee, J. Gu, J.-O Lee, K.-H. Yoo, H. Tanaka, H. Tabata, and T. Kawai, Jpn. J. Appl. Phys. 41, 891 (2002). 